\title{
AA.VV., Hacia un modelo de justicia restaurativa empresarial*
}

(2021) Seminario web

Instituto de Derecho Penal

Europeo e Internacional (UCLM)

\author{
Maite Carretero Sanjuan \\ Universidad Carlos III de Madrid \\ ORCID ID 0000-0001-6826-2072 \\ mtcarret@der-pu.uc3m.es
}

\section{Cita recomendada:}

Carretero Sanjuan, M. (2021). AA.VV., Hacia un modelo de justicia restaurativa empresarial. Eunomía. Revista en Cultura de la Legalidad, 21, pp. 483-490.

doi: https://doi.org/10.20318/eunomia.2021.6381

Recibido / received: 05/05/2021

\section{Resumen}

En el complejo y concreto escenario de comportamientos corporativos ilícitos que ocasionen o puedan ocasionar graves daños a los derechos humanos o al medio ambiente, este trabajo pretende sentar las bases de un modelo de justicia restaurativa empresarial que se presente como una suerte de directrices $u$ orientaciones en dinámicas restaurativas de gran complejidad. Sus resultados se corresponden con los análisis, debates y reflexiones de los diversos ponentes que participaron en el seminario «Hacia un modelo de justicia restaurativa empresarial», organizado por el Instituto de Derecho Penal Europeo e Internacional de la Universidad de Castilla-La Mancha en el marco del Proyecto REPMULT- Responsabilidad penal de empresas multinacionales por violaciones de derechos humanos y al medio ambiente.

\section{Palabras clave}

Justicia restaurativa, Conflicto, Delincuencia económica, Restauración, Mediación.

\footnotetext{
* Este trabajo forma parte del Proyecto de Investigación REPMULT, Responsabilidad penal de las empresas multinacionales por violaciones de derechos humanos y al medio ambiente. MINECO/AEI/FEDER DER2017-85144-C2.
} 


\begin{abstract}
In the complex and specific scenario of unlawful corporate behaviours that cause or may cause serious harm to human rights or to the environment, this work aims to lay the foundations of a model of corporate restorative justice that presents itself as a set of guidelines or orientations in highly complex restorative dynamics. Its results correspond to the analysis, debates, and reflections of the various speakers who took part in the seminar "Towards a model of corporate restorative justice" organised by the Institute of European and International Criminal Law of the University of Castilla-La Mancha within the framework of the REPMULT Project - Criminal liability of multinational companies for violations of human rights and the environment.
\end{abstract}

\title{
Keywords
}

Restorative justice, Conflict, Economic crime, Restoration, Mediation.

SUMARIO. 1. Introducción. 2. Problemas y retos de la justicia restaurativa empresarial. 3 . Operativización de la justicia restaurativa empresarial. 4. Los fondos fiduciarios como mecanismos de reparación. 5. Conclusiones.

\section{Nota preliminar}

En este trabajo se pretenden sintetizar los análisis, debates y conclusiones alcanzadas en el seminario web «Hacia un modelo de justicia restaurativa empresarial» ${ }^{1}$, organizado por el Instituto de Derecho Penal Europeo e Internacional de la Universidad de Castilla-La Mancha en el marco del Proyecto REPMULT Responsabilidad penal de empresas multinacionales por violaciones de derechos humanos y al medio ambiente.

Este Seminario acogió a ponentes de diversas nacionalidades que, durante dos jornadas, reflexionaron y debatieron, desde una perspectiva teórica y práctica, sobre la temática que aquí abordamos, con la pretensión de sentar las bases de un modelo operativo de justicia restaurativa empresarial que cristalice una alternativa real, eficiente y eficaz al modelo de justicia tradicional en el concreto ámbito de comportamientos corporativos ilícitos que ocasionen o puedan ocasionar graves daños a los derechos humanos o al medio ambiente.

\section{Introducción}

En el contexto actual, en el que los conflictos se presentan como cambiantes e inciertos, el Derecho tiene ciertas limitaciones para dar respuestas eficientes y eficaces a determinados conflictos, lo que deriva en la necesidad de contar con otras vías de gestión e intervención. En el ámbito concreto que nos ocupa, escenarios de conflictividad derivada de comportamientos corporativos ilícitos que ocasionen o puedan ocasionar graves daños a los derechos humanos o al medio ambiente, se afirma que el proceso penal tiene serias limitaciones, a saber, no atiende suficientemente a la reparación del daño, no está ideado para escuchar a las víctimas, se aleja de ciertas tipologías de víctimas y olvida la orientación preventivo-especial positiva en el delincuente económico.

Ante esta realidad, la justicia restaurativa se presenta como alternativa con un tremendo potencial en escenarios de conflictividad derivada de comportamientos

\footnotetext{
${ }^{1}$ La totalidad de las reflexiones y propuestas de este trabajo nacen en las intervenciones de los ponentes en el Seminario, siendo todo mérito atribuible a sus investigaciones. Por orden de intervención: Adán Nieto Martín, Roberta Guasti, Daniela Arantes, Beatriz López Lorca, Juan Ramón de Páramo, Raúl Calvo Soler, Eduardo Saad, Adriano Martufi y María José Guardiola.
} 
corporativos ilícitos con afección a derechos humanos o al medio ambiente. Siguiendo la definición dada por Naciones Unidas en su «Manual sobre programas de justicia restaurativa», se identifican varias características que conceptualizan este término:

- Supone un nuevo enfoque para la solución de problemas.

- Involucra a víctima, ofensor, instituciones sociales y comunidad.

- Se basa en el reconocimiento del daño a víctimas y comunidad.

- Comprende un esfuerzo por proporcionar ayuda y apoyo a víctima y victimario.

- Requiere de la responsabilización del victimario.

- Enfatiza la construcción de relaciones y reconciliaciones.

- Su foco está, más allá del pasado y del presente, en el futuro.

- Comprende un proceso de diálogo.

Una conceptualización clara del término lleva a diferenciarlo de otros que han podido mezclarse o confundirse con este. En este orden de ideas es preciso distinguir lo extrajudicial de lo restaurativo, entendiendo que ni todo lo restaurativo es extrajudicial ni todo lo extrajudicial es restaurativo. Tampoco la justicia restaurativa agota lo restaurativo, entendido como una forma de pensar y entender las relaciones humanas, ni la justicia restaurativa se agota en el discurso del Derecho penal restaurativo, aunque, por su propia idiosincrasia, sea este el ámbito en el que más impacto ha tenido el discurso restaurativo.

\section{Problemas y retos de la justicia restaurativa empresarial}

En las sesiones del Seminario se identificó un reto general de la justicia restaurativa empresarial que descansa en la necesidad de superar determinadas objeciones que, tradicionalmente, se han presentado sobre esta. Algunas de estas objeciones se resumen en que la justicia restaurativa no ha sido pensada para este tipo de conflictos y en que es una suerte de «justicia blanda». Así, se defiende que, aunque, efectivamente, la justicia restaurativa no se ha pensado en un contexto de comportamientos organizacionales ilícitos, ello no se presenta como un argumento que deba impedir que se puedan ir elaborando nuevas construcciones teóricas que respondan a las potenciales problemáticas por el camino. La idea de que se trata de un tipo de «justicia blanda», a pesar de haber sido recurrente, es debatida defendiendo que es muy cuestionable, pues para el delincuente económico, se afirma, puede resultar más sencillo y atractivo el proceso penal de lo que lo es reconocer al otro, reconocer el daño ocasionado y enfrentarse a sus resultados.

En un nivel más operativo, se identificaron otra serie de problemas potenciales o retos a la hora de avanzar en el terreno de la justicia restaurativa empresarial. El primero, de índole organizativo o burocrático, se traduce en cómo se puede organizar un sistema capaz de hacer frente a daños de magnitudes tan relevantes como los derivados del escenario planteado, en los que identificación y participación de las partes presentan dificultades añadidas y las finalidades perseguidas son igual de necesarias que de ambiciosas. Un segundo bloque radicaría en la necesidad de concretar qué rasgos debe tener un procedimiento para poder afirmar que es restaurativo. Y un último bloque se referiría a cómo se debe estructurar la relación entre el proceso restaurativo y el eventual proceso penal. A todas ellas nos referiremos en esta exposición.

\section{Operativización de la justicia restaurativa empresarial}

Ante una meta tan ambiciosa, como ante cualquier problema complejo, se requiere identificar sus diferentes aristas en orden a ir componiendo, desde lo específico, lo 
general. En este proceso, se afirma, lo primero sería un cambio de las máximas de la justicia tradicional o clásica a las de la justicia realmente restaurativa. Así, se propone pasar del caso al conflicto. El caso no sería sino una reducción del conflicto que incorpora ciertas propiedades de este que pueden no ser las relevantes para su comprensión. Resolver el caso no implica resolver el conflicto y, en los supuestos que nos ocupan, se defiende que la reducción al caso tiende a ser muy ineficiente.

El segundo cambio propuesto sería del concepto de culpa al de daño y afectación de las relaciones entre las personas y las instituciones. La construcción del daño aquí iría mucho más allá de su entendimiento como afectación a un bien jurídico protegido.

El tercero lleva a exaltar el «diálogo» frente al «modelo de monólogos» que tradicionalmente ha impregnado la justicia penal. El proceso restaurativo se basa en las máximas de cooperar, colaborar y componer. La solución de la conflictividad se presenta como resultado de la interacción dialogada entre las partes, dentro de la cual aparecen los conceptos de reconocimiento y responsabilización a los efectos de que el victimario pueda hacerse responsable de sus acciones, de lo acontecido.

El cuarto cambio de enfoque que se propone supone pasar del concepto de «parte» al de "participante en un conflicto». Las personas involucradas serían protagonistas en la definición del conflicto, en la identificación del relato, en la concreción del daño y en la propuesta de solución y serían tratadas, más que como portadoras de intereses jurídicos, como seres humanos dotados de un patrimonio afectivo y emocional.

Por último, se afirma la necesidad de centrar el proceso en la «restauración» frente a la «reparación o indemnización del daño». Restaurar se presenta como ir un paso más allá, como atender a daños tangibles e intangibles y poner el foco en el discurso del daño y de la relación. Se defiende que un proceso que agota la relación en el abono del daño no es restaurativo y que se debe dar cuenta de la recomposición de los vínculos que se vieron afectados por el comportamiento ilícito. En definitiva, se afirma, la reparación restaura económicamente sin resolver el conflicto entre víctima y victimario y sin prevenir nuevas vulneraciones, manifestándose, así, como necesaria la restauración.

Tras este cambio de enfoque, se propone realizar una serie de consideraciones respecto a víctima, victimario y comunidad en el contexto concreto de ilícitos corporativos de grandes magnitudes. En estos, la víctima suele ser colectiva $y$, dentro de esa colectividad, hay distintas tipologías de victimización, a cuyas particularidades habrá que atender en el diseño de la estrategia restaurativa. Se afirma que es habitual que el daño, aquí, trascienda de las víctimas individuales, afectando, de algún modo, a la comunidad y sin olvidar que, a veces, ante víctimas difusas, la comunidad es la víctima y la víctima es la comunidad.

Se señala, como esencial en la consideración de la víctima en un proceso restaurativo, que ésta se encuentra con una posible situación de asimetría respecto al victimario. En los casos en los que el Seminario se centra, el victimario suele ser una gran multinacional frente a una población vulnerable y con escasos medios para clamar. El objetivo del proceso restaurativo, en este punto, sería combatir la asimetría de poder, consiguiendo que el acuerdo sea un reflejo real de la voluntad de las partes en un contexto de simetría e igualdad.

Los intervinientes en el Seminario identificaron, asimismo, que esta relación de asimetría se puede ver elevada a una situación de absoluta dependencia en la que 
las víctimas sufren una doble victimización, primero, por el comportamiento organizacional y, después, por la sanción a la empresa derivada de ese mismo comportamiento. Se refieren, así, a casos en los que la supervivencia económica y social de una comunidad depende del victimario, que asume un doble rol de ofensor y salvador. En estos, se afirma, la justicia restaurativa es aún más importante, en aras a conseguir la reconstrucción efectiva de la vida de las personas que se han visto afectadas por el ilícito.

Respecto del victimario, quizás, la cuestión más relevante sea la necesidad de motivarles, a través de estímulos o incentivos, para que voluntariamente opten por participar en un proceso restaurativo. El incentivo clave que se identifica, en este sentido, es la mejora de su imagen pública. El principal riesgo identificado deviene del entendimiento de la responsabilización en el proceso restaurativo como una admisión de la culpa en la lógica judicial. Es clave, se dice, ante este riesgo, entender que el proceso de construcción de reconocimiento y responsabilización no se agota ni equivale al proceso de reconocimiento de la culpa en la lógica de la estrategia judicial.

En esta delimitación de víctimas y victimarios el Seminario precisa que no todo el que quiera participar en el proceso restaurativo estará autorizado para hacerlo. En una primera aproximación, el criterio que se propone, a modo de filtro, es requerir una clara orientación restaurativa. Se precisa un enfoque específico que evite que, en el proceso restaurativo, haya víctimas o victimarios vindicativos.

La relación entre el proceso restaurativo y el proceso penal se revela en los debates como uno de los grandes retos a los que se enfrenta esta meta de operativizar la justicia restaurativa empresarial. Como modelos-tipo se identifican distintos escenarios en esta relación. Es posible entender que se trata de procesos diferentes que, por atender a finalidades distintas, deben caminar en paralelo. Se propone, de otro lado, pensar en ambos procesos como alternativos o verlos desde una óptica de interrelación. La autenticidad, credibilidad y eficacia de los resultados de un programa restaurativo tienden a variar según el modelo por el que se opte.

Desde la lógica de una relación alternativa, en un determinado momento, el proceso judicial derivaría el caso al proceso restaurativo, entendiendo que este puede resolver mejor el conflicto y asumiendo, de algún modo, los resultados que se alcancen en las dinámicas restaurativas.

En un escenario de interrelación, en el que ambos procesos fuesen a la par y estuviesen, en cierto modo, conectados, se propone pensar en esa conexión a través de la atenuante de reparación o de una sentencia de conformidad que incorporase los resultados alcanzados en fase restaurativa, entendiendo que la restauración puede afectar a la necesidad de la pena. Aquí, se afirma, deben evitarse efectos automáticos que pueden instrumentalizar la justicia restaurativa o que afecten a la necesaria voluntariedad de estos procesos.

En una versión máxima, más atrevida, se llegó a plantear un cuarto escenario en el que la justicia restaurativa ocuparía la totalidad del espacio penal en cualquier dinámica. Se trataría de construir, desde cero, una justicia penal exclusivamente restaurativa, abandonando cualquier conceptualización de la pena y del proceso en una lógica retributiva o reparadora. Se afirma que, quizás, no nos paremos a reflexionar sobre esta posibilidad porque partimos de un Derecho penal que es cómo es y sobre el que pretendemos acciones de mejora. En cualquier caso, el Seminario añade que el problema aquí, quizás, sea que no estamos preparados para un cambio así. Se defiende que la voluntariedad necesaria en un proceso restaurativo obliga a llevar por la vía judicial tradicional aquellos casos en los que en víctima y/o victimario 
no concurra y que hay casos que, por su propia idiosincrasia, pueden no ser adecuados para procesos restaurativos, y pueden requerir de la lógica del proceso formal.

En este punto, resulta conveniente hacer una breve mención a dos cuestiones transversales tratadas en el Seminario. De un lado, a la mediación como instrumento de la justicia restaurativa. De otro, a los fondos fiduciarios como mecanismos de reparación, cuestión esta que, por su alcance, será tratada en un apartado diferenciado.

Se afirma que una primera aproximación a la mediación requiere, precisamente, identificarla como un útil de la justicia restaurativa, pues, a pesar de que en más de una ocasión ambos términos se han considerado como sinónimos, la justicia restaurativa no se reduce ni puede reducirse a la mediación. Dicho esto, como instrumento, la mediación tiene una técnica bastante elaborada que puede resultar muy útil en procesos restaurativos.

Tradicionalmente, se ha hecho referencia a tres grandes modelos de mediación, con sus variantes y actualizaciones, diferentes entre sí, pero también complementarios en una visión tridimensional de los problemas: el Modelo de Harvard, en una línea estratégica-analítica; el Modelo Transformativo, desde una línea más orientada a la psicología social transformativa; y el Modelo Narrativo o Circular Narrativo, en una línea más narrativa. Respecto a la pregunta qué modelo sería óptimo para los conflictos que nos ocupan, los intervinientes concluyeron que no existe una respuesta aplicable a todo conflicto, sino que habrá que atender a las concretas particularidades de cada conflicto, a la fase concreta del proceso restaurativo en que se encuentre o a la finalidad específica que se pretenda, dentro de la multiplicidad de finalidades perseguidas por la justicia restaurativa, para determinar la idoneidad de la aplicación de un modelo o de la aplicación simultánea o sucesiva de varios modelos. Se afirma la necesidad de prever, además, la posibilidad de que la mediación no deba formar parte del diseño del proceso restaurativo en determinados casos. Así, podría no ser la opción recomendable ante conflictos con multitud de participantes o en los que no fuese posible garantizar una relación simétrica entre éstos. Se concluye, así, que lo adecuado sería realizar un concreto diagnóstico de la conflictividad que dé la información suficiente como para desarrollar, del mejor modo posible, una estrategia restaurativa.

\section{Los fondos fiduciarios como mecanismos de reparación}

Es evidente que la intersección entre la actividad empresarial y los derechos humanos se tiene que materializar en una fase de responsabilidad. Lo que no es tan evidente es la modalidad de responsabilidad que se le puede exigir a la empresa, cómo puede concretarse y ante qué comportamientos.

En este contexto, los fondos fiduciarios, como una suerte de mecanismos financieros orientados a la reparación, han pasado relativamente desapercibidos. Se afirma que no han sido todo lo visibles que debieran en el marco de la justicia restaurativa y ello a pesar de ser un instrumento convenientemente orientado a la reparación del daño y con potencial para ser un gran elemento restaurativo.

Es habitual asociar los fondos fiduciarios a consecuencias de procesos civiles o penales, pero también pueden derivar de una labor de arbitraje o mediación, presentando mayor versatilidad. 
La flexibilidad de los fondos fiduciarios permite la corresponsabilidad entre Estado y empresas. En este escenario, se identifican dos grandes modelos de fondos fiduciarios, a saber, fondos en los que la participación mayoritaria es del Estado con organizaciones internacionales y fondos en los que únicamente intervienen empresas. El modelo que se defiende como más interesante en un contexto como el que nos ocupa, no obstante, sería un modelo mixto en el que participaran por igual empresas y Estado, mediante donaciones fijas o a través de contribuciones. Ello, se afirma, permitiría lograr la necesaria corresponsabilidad a la que se hacía mención.

Se defiende que la gran versatilidad de estos mecanismos financieros permite su orientación a la reparación en un doble sentido reactivo y proactivo. En la dimensión de carácter preventivo, los fondos fiduciarios permiten introducir acciones de reacción temprana (como cambios activos en la propia empresa) y entender el daño desde una perspectiva multidimensional.

Aún sin haber cobrado el protagonismo que debieran, estos mecanismos han sido utilizados en casos de gran trascendencia como la ruptura del pozo Macondo de British Petroleum en el Golfo de México o el derrumbe del Rana Plaza en Bangladesh. Se afirma que la experiencia, en estos casos, muestra que los fondos fiduciarios pueden tener una gran versatilidad en la práctica y una gran efectividad en la reparación del daño. No obstante, también se señala que éstos se enfrentan, a pesar de ser un mecanismo financiero en sentido estricto, a algunos de los desafíos mencionados para la justicia restaurativa, tales como la determinación del daño, las características que debe reunir para ser eficaz o la necesidad de identificar a las víctimas.

\section{Conclusiones}

En estas sesiones, pues, se concluye que la justicia restaurativa puede y debe resultar aplicable a escenarios de conflictividad derivada de comportamientos corporativos ilícitos que ocasionen o puedan ocasionar graves daños a los derechos humanos o al medio ambiente. Es un terreno muy incipiente, con un extraordinario potencial, en el que aún queda mucha reflexión y debate.

Se defiende asimismo que el proceso de operativización de la justicia restaurativa empresarial se inicia con un cambio de enfoque: pasar del caso al conflicto, de la culpa al daño de la relación, del monólogo al diálogo, de la determinación clásica del daño por el juez a un discurso de composición, de participantes a protagonistas, de reparar e indemnizar a restaurar, centrando la atención en el reconocimiento y la responsabilización del victimario tanto como en la idea de la legitimación de la víctima.

En esta intencionalidad de operativizar la justicia restaurativa empresarial se evidencia la dificultad derivada de las posibles interacciones entre proceso penal y proceso restaurativo cuando ambos coexisten. Más allá de la concreción del modelo de interacción adecuado en cada contexto y en cada conflicto, se defiende la necesidad, en líneas generales, de un Derecho más flexible y responsable, que se adapte a la realidad actual de los conflictos sociales entendidos como complejos, cambiantes e inciertos.

En casos de interrelación entre proceso restaurativo y proceso formal, configurados como una suerte de suspensión del proceso o de la ejecución de la pena, se plantea la problemática de qué ocurre si el proceso restaurativo falla y, sin duda, el proceso restaurativo puede fallar, como también, se afirma, falla el proceso penal cuando no cumple sus finalidades retributivas, preventivo generales 0 
preventivo especiales. Se defiende que cuando el proceso penal falla, no hacemos sino volver al mismo sistema para buscar, de nuevo, una solución para el caso. Entonces, ¿por qué en el proceso restaurativo ese fallo debe determinar el abandono de las pretensiones restaurativas y el retorno al proceso penal?

Asimismo, se puso de manifiesto que, en ocasiones, se pide al discurso restaurativo que responda a preguntas que el discurso no restaurativo tampoco ha logrado resolver y que ésta no parece una buena estrategia. Qué se pide y qué se espera del discurso restaurativo debe concretarse desde la coherencia, en términos más humildes y alcanzables. Se concluye que la justicia restaurativa no siempre tiene que triunfar como tampoco tiene que hacerlo el Derecho penal.

Mucho se ha discutido sobre la idoneidad o no de las diferentes técnicas y modelos en la gestión y resolución de conflictos y, al final, lo que se concluye es que esa adecuación o idoneidad depende del tipo de conflicto. Lo relevante, entonces, sería diagnosticar de forma adecuada las características sobre las que se va a trabajar para pronosticar de la mejor manera posible cuál es la mejor herramienta dadas esas características.

Finalmente, se concluyó que el fondo fiduciario, revelado como un mecanismo con un extraordinario potencial, debe estar previsto como medio de reparación, ya sea de carácter estatal o empresarial. Se defiende que no debe ser concebido nunca desde la perspectiva de una sanción, pues perdería todo su potencial restaurativo. Si bien no se han producido hasta la fecha encuentros restaurativos en las dinámicas de los fondos fiduciarios, se afirma que no parece haber inconveniente, precisamente por la gran versatilidad de estos, para combinarlos con procesos restaurativos en sentido amplio. Así, los fondos fiduciarios se presentan como una gran alternativa en este contexto si se aprovechan en toda la extensión de una filosofía restaurativa.

Enlace a las sesiones del seminario sobre.Justicia Restaurativa Empresarial:

Cumplimiento Normativo UCLM. (2021, 9 febrero). SEMINARIO WEB: Experiencias restaurativas [Archivo de vídeo]. Recuperado de https://www.youtube.com/watch?v=quyyNgJLmCA\&ab channel=CumplimientoNorm $\underline{\text { ativoUCLM }}$

Cumplimiento Normativo UCLM. (2021b, 9 febrero). SEMINARIO WEB: Hacia un modelo de justicia restaurativa [Archivo de vídeo]. Recuperado de https://www.youtube.com/watch?v=MqZ2O8ShoA\&ab channel=CumplimientoNormativoUCLM 